\title{
Multispectral Imaging of Degraded Parchment
}

\author{
Lindsay MacDonald ${ }^{1}$, Alejandro Giacometti ${ }^{1}$, Alberto Campagnolo ${ }^{2}$, \\ Stuart Robson ${ }^{3}$, Tim Weyrich ${ }^{4}$, Melissa Terras ${ }^{5}$, and Adam Gibson ${ }^{1}$ \\ ${ }^{1}$ Dept. of Medical Physics \& Bioengineering, Faculty of Engineering, \\ University College London \\ \{lindsay.macdonald, alejandro.giacometti.09, agibson\} @ucl.ac.uk \\ ${ }^{2}$ Ligatus Research Centre, Camberwell College of Art, University of the Arts London \\ alberto. campagnolo@gmail.com \\ ${ }^{3}$ Dept. of Geomatic Engineering, University College London \\ srobsonacege.ucl.ac.uk \\ ${ }^{4}$ Department of Computer Science, University College London \\ t.weyrich@ucl.ac.uk \\ ${ }^{5}$ Centre for Digital Humanities, University College London \\ m.terras@ucl.ac.uk
}

\begin{abstract}
Multispectral imaging was employed to collect data on the degradation of an $18^{\text {th }}$ century parchment by a series of physical and chemical treatments. Each sample was photographed before and after treatment by a monochrome digital camera with 21 narrow-band filters, with the objective of assessing algorithms for recovery of text from the images. A template-matching technique was used to detect the circular holes in each sample and a four-point projective transform to register the 21 images. Colour accuracy was verified by comparison of reconstructed spectra with measurements by spectrophotometer.
\end{abstract}

Keywords: multispectral imaging, manuscript, spectral analysis, parchment.

\section{Introduction}

Digital surrogates of documents have become an indispensable tool for scholars and archives. They provide an easily accessible, non-degrading resource for research and study [1]. Numerous imaging and image processing technologies have been employed to examine the characteristics of the originals, such as X-ray imaging, imaging in the infrared spectrum, and multi-spectral imaging. These offer additional information about the physical characteristics and condition of the document. The results have been better images, more possibilities for analysis, and additional information recovered from ancient documents [2-4].

Large collections of parchment documents exist in public and private libraries, archives, and museums in varying degrees of preservation. Parchment has a predominantly organic composition, based on protein collagen in association with small quantities of elastin, globular proteins, and fats. It is prepared from an animal skin that has been wetted, immersed in lime water, dehaired, scraped, then left to dry under tension on a wooden frame. The stretching of the soaked pelt has the effect of reorganising the collagen fibre network into a laminate structure. The resulting 
material is a fairly stiff sheet which, without any further treatment, is durable and can last for centuries, provided it is kept cool and dry [5-7].

Digitisation projects generally concentrate on documents in their existing state. Typically the focus of image processing is to visualise the supposed original state of the document, before the damage occurred. Current 'best practice guidelines' for digitisation of 2D manuscript material (such as used by the Library of Congress) do not cover any aspect of multispectral capture or of calibration issues. Indeed most multispectral image capture projects of documentary material are one-off exploratory projects with an empirical approach. More structured research methods can encourage understanding and best practice approaches for this kind of documentary imaging.

In this project, we aimed to investigate the processes of controlled document degradation using multispectral imaging. We obtained an 18th-century manuscript deaccessioned from the London Metropolitan Archives. Although it was in good physical condition, this manuscript was a tedious legal contract, deemed to hold no historical value, and it was kindly donated for experimentation. It comprised two large sheets of prepared animal skin, i.e. parchment, written in iron gall ink and highlighted in red ink. Each sheet measured approximately $70 \times 70 \mathrm{~cm}$ (Fig. 1), and the two had been tightly folded together for storage. The document was an indenture (contract) between Mr John Sherman and Mr Christ Gardiner dated 11th August 1753.
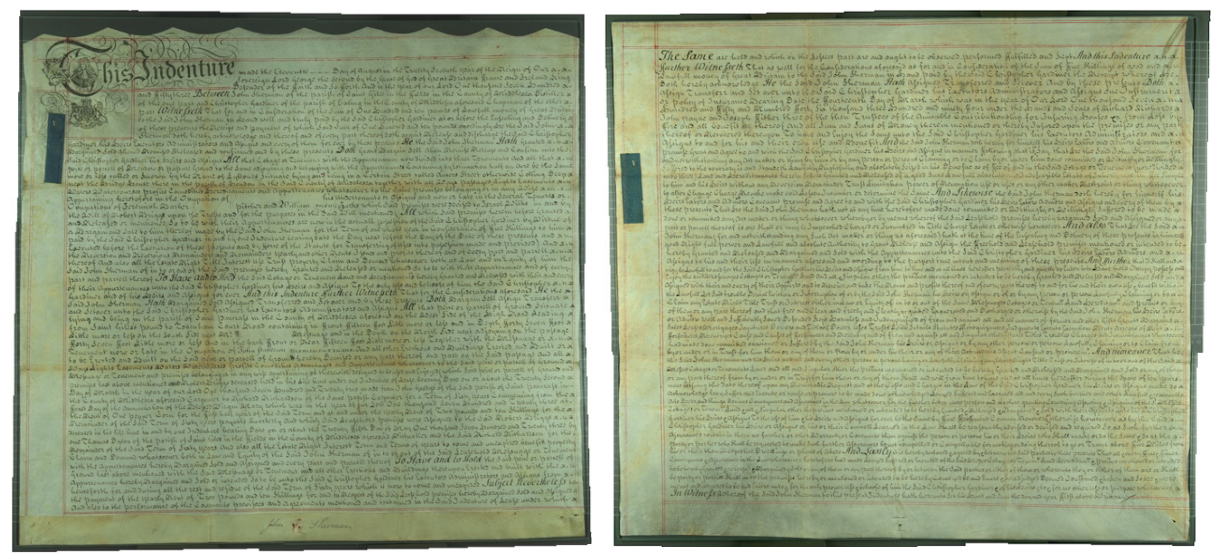

Fig. 1. Outer and inner sheets of complete manuscript, writing in Iron Gall ink on parchment

Parchment is an extremely hygroscopic material that reacts readily to changes of humidity with changes in thickness and physical appearance, such as cockling and curling. We selected various methods of artificial degradation for our samples to simulate the kinds of damage that frequently affect parchment documents found in cultural heritage collections. These degradation methods fall within the main categories of mechanical, thermochemical, humidity, biological, and physical damage, all of which act directly upon the writing medium, changing its physical characteristics at both microscopic and macroscopic levels.

Twenty-three square sections of $8 \times 8 \mathrm{~cm}$ were cut from the original manuscript, with each section containing written text. Two sections had text written on both sides. Each sample was exposed to an external deteriorating agent to produce a controlled 
degradation, including mechanical damage, heat, humidity, abrasion and a variety of substances with different chemical properties, such as acid, alkaline, bleach, tea and human blood. These affected the appearance and condition of the samples in different ways, typical of the actual damage suffered by parchment in real archives. Preliminary tests proved the viability of the procedures for degrading the samples and enabled parameters to be established for each treatment process [8].

\section{$2 \quad$ Multispectral Imaging}

A multispectral image is a set of monochrome images acquired through narrow band filters for consecutive wavebands. Each image shows the intensity of radiation from the scene in the corresponding waveband [9]. Images are acquired at visible wavelengths (400-700 $\mathrm{nm})$, and may also include regions of the non-visible spectrum: ultraviolet $(<400 \mathrm{~nm})$ and infrared $(>700 \mathrm{~nm})$. Multispectral image capture was originally developed for remote sensing to determine the composition of objects in space by measuring their emission spectra. NASA satellites were also designed to capture multi-spectral reflective images from Earth, the most famous being Landsat, first launched in 1972, with eight spectral bands. The original application was to identify geographic resources without the need for high spatial image resolution [10].

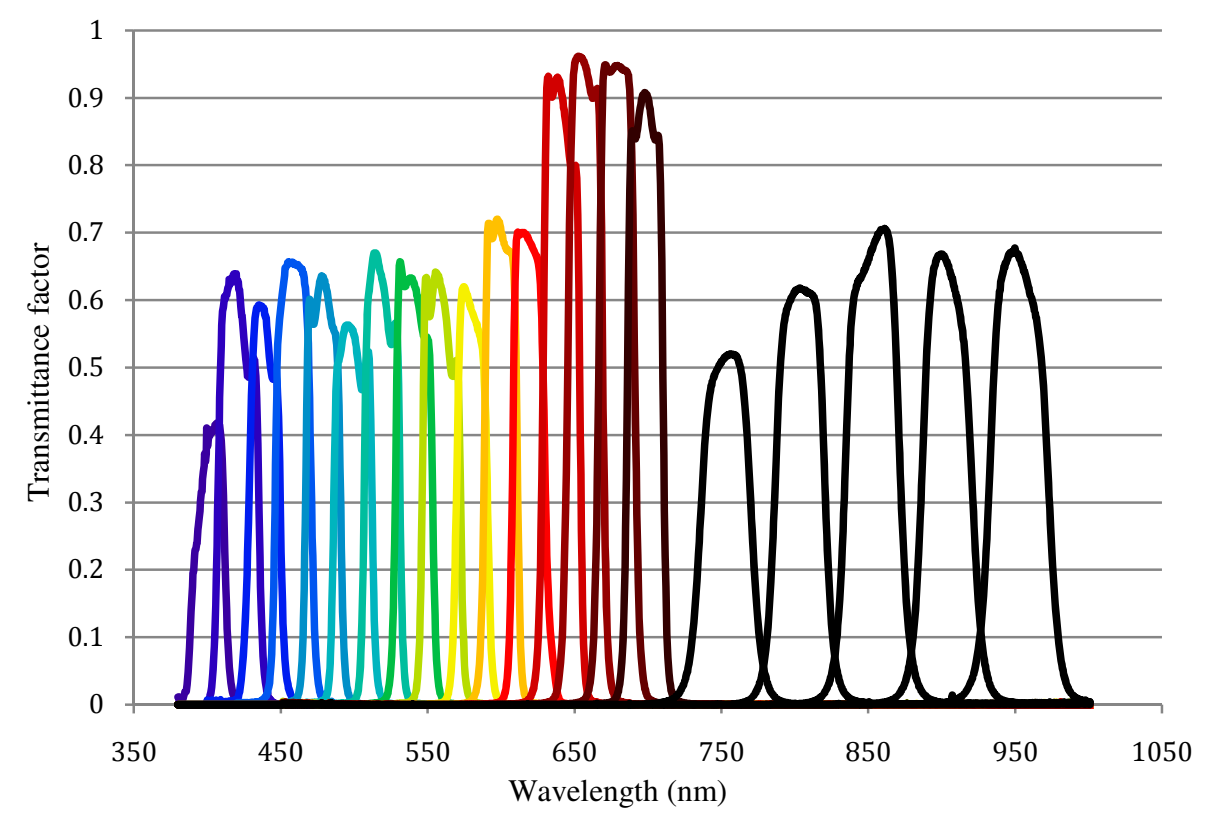

Fig. 2. Transmittance factors of 21 optical bandpass filters in the visible and NIR spectrum

Multispectral imaging has been successfully applied to fine art paintings [11-12], ancient documents [3], and other cultural heritage applications [13-14]. Bearman and Spiro investigated the Dead Sea Scrolls, with a liquid crystal tunable filter in front of a monochrome CCD camera [15]. They captured digital images from 400 to $1050 \mathrm{~nm}$, and noted a great improvement in legibility at $970 \mathrm{~nm}$, compared with $700 \mathrm{~nm}$. 
Multispectral images can be used to discriminate between reflectance spectra, and hence to identify and separate different types of inks in a single document, showing whether the document has been edited. The spectral reflectance profiles of known materials can be stored in a database and used to classify unknown materials. The type of ink used in manuscripts has considerable impact on the imaging approach. The earlier carbon black ink consisted of graphite or soot particles suspended in an organic binder and applied with a stylus. Such inks do not penetrate the parchment but rest on top, with particles adhering to the micro-structure of the surface. Iron gall ink, also known as gallo-tannin ink, was introduced around the third century AD, prepared with organic material that penetrates more deeply into the substrate and reacts, staining it black. Chabries et al noted that parchment reflectance increases at longer wavelengths, resulting in greater contrast of text in infrared images [2].

Each of our parchment samples was imaged before and after the treatment, using two different cameras, in combination with two lighting systems for both reflective and transmissive imaging, through a series of bandpass filters. The cameras were: (1) Nikon D200 with $105 \mathrm{~mm} \mathrm{f/2.8} \mathrm{lens,} \mathrm{a} \mathrm{digital} \mathrm{SLR} \mathrm{which} \mathrm{captures} \mathrm{RGB} \mathrm{images} \mathrm{of}$ $3900 x 2600$ pixels in the visible spectrum; (2) Kodak Megaplus 1.6i scientific camera with Nikkor $50 \mathrm{~mm} \mathrm{f} / 2$ lens, which captures monochrome images of 1536x1024 pixels in both the visible and near-infrared spectrum over the range $400-1100 \mathrm{~nm}$. For the Nikon camera, a set of 16 bandpass optical interference filters was used, spaced at regular intervals across the visible spectrum. For the Kodak camera 5 additional filters were used to cover the near-infra-red range.

Each of the first 16 filters had a bandwidth of approximately $20 \mathrm{~nm}$, at wavelength intervals of $20 \mathrm{~nm}$ throughout the visible spectrum from 400 to $700 \mathrm{~nm}$ inclusive. The last 5 filters had a bandwidth of approximately $50 \mathrm{~nm}$, at intervals of $50 \mathrm{~nm}$ in the

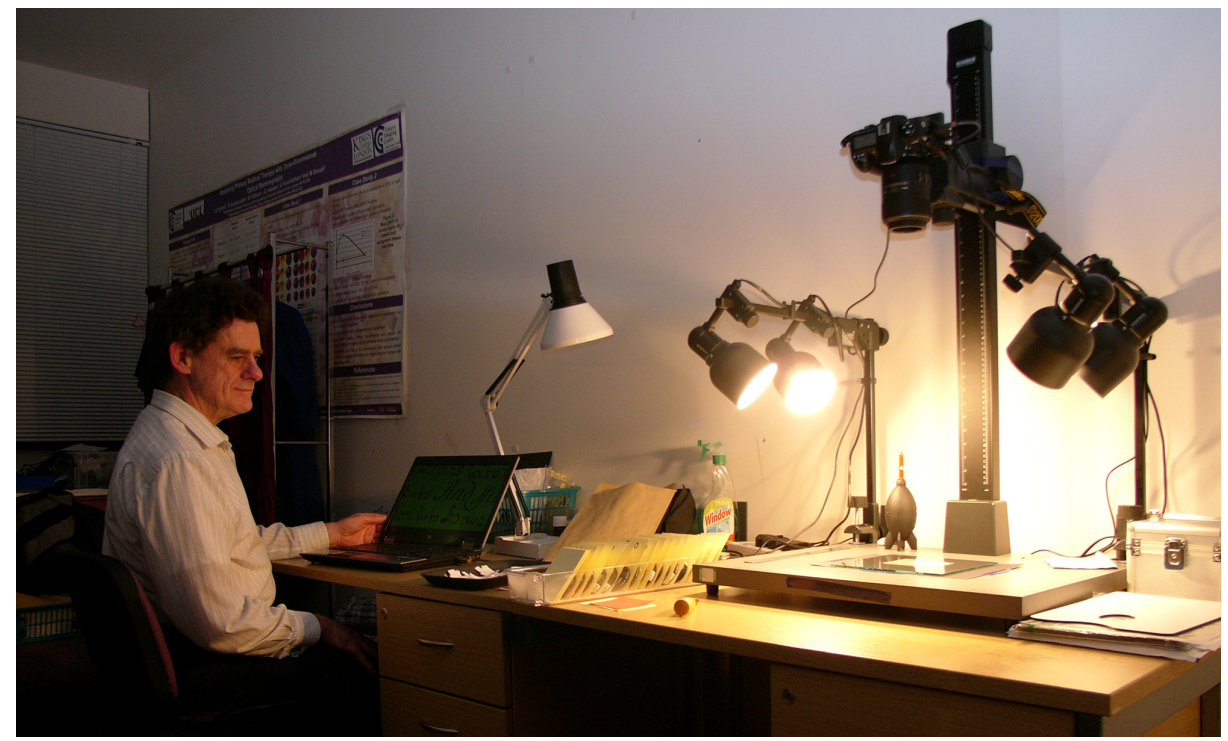

Fig. 3. Copystand with four tungsten-halogen lamps for reflective imaging of samples with the Nikon D200 camera. The filter is screw-mounted into the front of the lens. 
near-infrared spectrum from 750 to $950 \mathrm{~nm}$ inclusive. All were circular glass filters in $52 \mathrm{~mm}$ rings for screw-mounting onto the front of the lens of each camera. This arrangement guaranteed that no stray light entered the lens. The spectral transmittance of each filter was measured with an Ocean Optics HR2000+ spectrometer (Fig. 2).

The samples were illuminated by four tungsten-halogen lamps on a standard photographic document copystand (Fig. 3). This setup with an incident angle of approximately $45^{\circ}$ allowed the camera to capture light reflected from the parchment without specular highlights. Because parchment is prone to curl, a $3 \mathrm{~mm}$ glass plate with anti-reflective coating was placed over the sample to hold it flat on the baseboard. In total $23 \times 21 \times 2=966$ images were captured by the Kodak camera for parchment samples before and after treatment, and a further $23 \times 16 \times 2=736$ images for the Nikon camera.

Four circular holes of diameter $1 \mathrm{~mm}$ were drilled in each sample at approximately one third and two thirds of the width and height. These holes are apparent in each image and remained as persistent features after the samples were degraded, both as reference points for registration of the multispectral image channels and for comparison of image sets of the samples before and after treatment.

\section{Calibration}

The discussion in this article focuses on the Kodak Megaplus camera. Although its image resolution is relatively low (image size $1536 \times 1024$ pixels), its large photosite area on the CCD sensor and absence of a colour filter array and an infrared cut-off filter gives it good linearity and signal-to-noise performance throughout the whole spectral range. The lens was set to $f / 5.6$, and the exposure time varied for each filter so that the white level was just below the CCD saturation level over the whole image.

The spectral power distribution of the tungsten-halogen lamps was measured with the Ocean Optics spectrometer over the range 200 to $1100 \mathrm{~nm}$, at intervals of approximately $0.5 \mathrm{~nm}$ (Fig. 16). The spectrum was continuous between 400 and 1000 $\mathrm{nm}$, with a broad peak between 580 and $680 \mathrm{~nm}$. For wavelengths shorter than 440 $\mathrm{nm}$ and longer than $920 \mathrm{~nm}$ there was insufficient power to achieve reliable results.
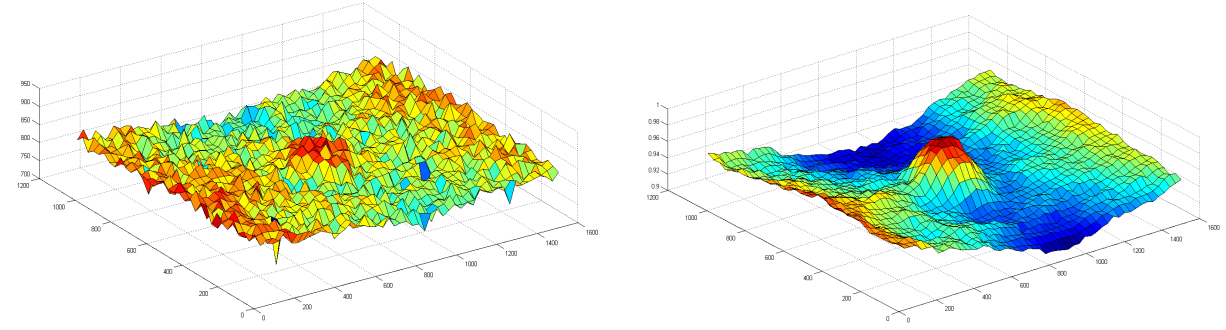

Fig. 4. Illumination intensity distribution for raw (left) and smoothed (right) images

A stack of 10 sheets of white paper was used to determine the non-uniform distribution of illumination. The captured image of the white paper was very irregular (Fig. 4a), caused by both camera noise and texture of the paper. A box mean filter 
with a square kernel of $31 \times 31$ pixels was applied to smooth the illumination profile (Fig. 4b). The black level of the camera (set by thermal noise and ADC voltage offset) was determined by capturing a series of images with the lens cap on. The mean over 70 images with exposure times ranging from 10 to $700 \mathrm{msec}$ was 27.1 on the 10-bit scale 0-1023 (Fig. 6a), with a standard deviation of 1.8. There was no significant variation with exposure time or position in the image.
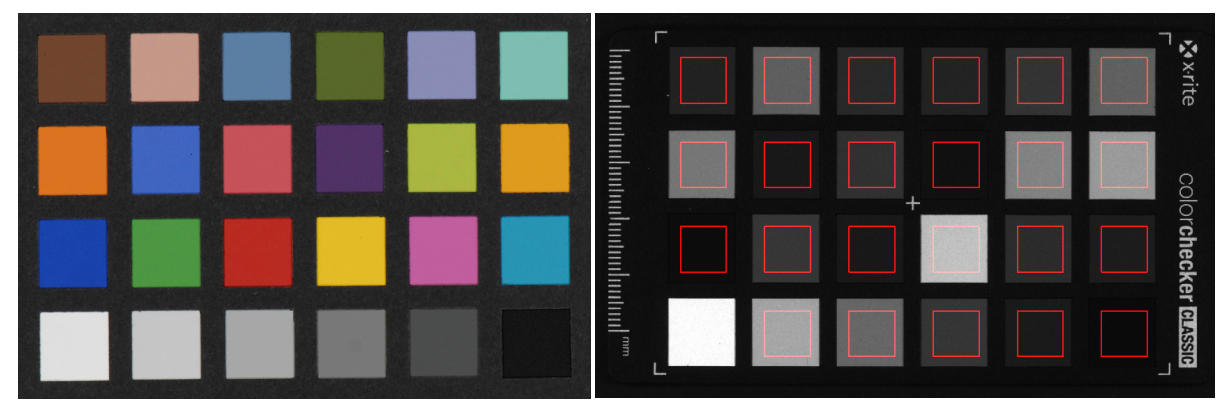

Fig. 5. (left) Colour image of MiniMacbeth target taken by Nikon D200 (no filter) under flash illumination; (right) Monochrome image of target taken with Kodak camera through $580 \mathrm{~nm}$ filter, showing position of square sampling box for each patch (red outlines)

The tonal linearity and colour accuracy were analysed with the aid of a MiniMacbeth Color Checker target (Fig. 5a). Twenty-one images were taken through the successive filters, using the same camera settings as for the parchment samples. The value for each patch was computed as the average of an area of 100x100 pixels in the image (Fig. 5b), using the correction formula:

$$
\hat{I}_{k}=\frac{I_{k}-B}{\left(W_{k} / r_{k}\right)-B}
$$

where for waveband $k: \hat{I}_{k}$ is the corrected and normalised image, $I_{k}$ is the original image, $W_{k}$ is the smoothed white illumination profile image, $B$ is the smoothed black image, and $r_{k}$ is the reflectance factor of the white paper.
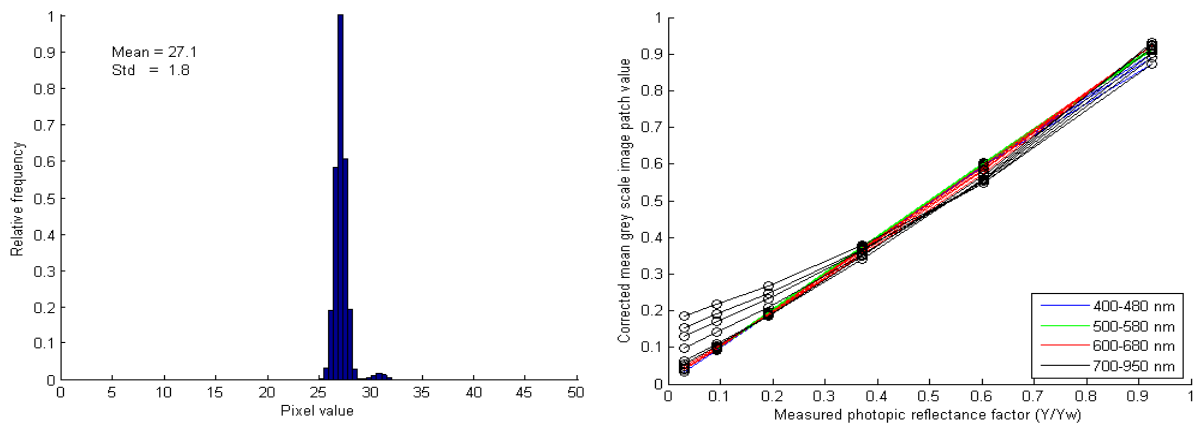

Fig. 6. (left) Histogram of black values for Kodak camera, averaged over 70 exposures; (right) Grey-scale tone reproduction curve for all filter wavelengths 
The reflectance spectrum of the white paper was measured with an X-Rite i1Pro spectrophotometer (Fig. 7a) and shows a peak $>1$ at a wavelength of approximately $435 \mathrm{~nm}$, caused by the fluorescent whitening agent in the paper. A notable feature was that the reflectance of the masking grid between the patches, which looks black in the visible spectrum, rose rapidly in the infrared, becoming a light grey at $950 \mathrm{~nm}$. The black patch in the bottom right corner, however, was much more stable because it is made with a carbon-based pigment and remains low in reflectance at all wavelengths.
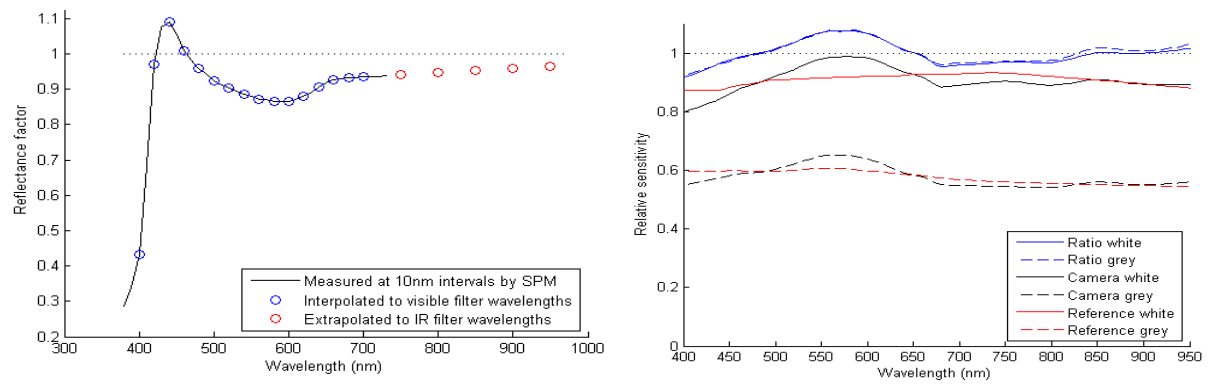

Fig. 7. (left) Spectral reflectance factor of white paper used for illumination profile; (right) camera sensitivity vs wavelength (blue lines) inferred from white and grey patches on target

The mean patch values for the grey scale (six patches on the bottom row of the target) were plotted against the measured relative luminance values (Fig. 7b). The tonal linearity was excellent for the visible wavelengths, but departed significantly at the dark end for infrared wavelengths because of lens flare and loss of image contrast.
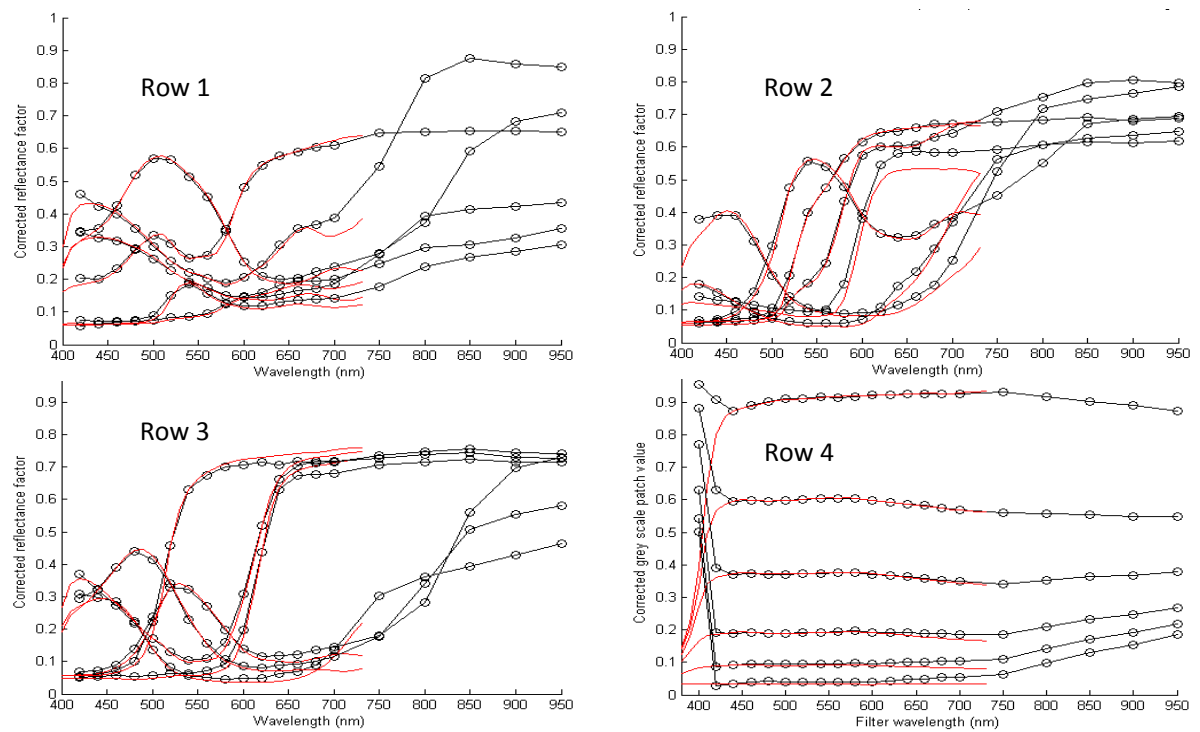

Fig. 8. Reflectance factor vs wavelength for camera signals (black lines with circles) and spectrophotometer measurements (red lines) for four rows of the MiniMacbeth target 
The spectral sensitivity of the camera was inferred from the grey scale for each wavelength by comparing the camera response with the measured reflectance. The reflectance at the centre wavelengths of the five IR filters beyond $730 \mathrm{~nm}$ was extrapolated from measurements with the ilPro spectrophotometer. The ratios for the white and light grey patches yielded very similar results (blue lines in Fig. 7b).

To check the accuracy of reconstruction of reflectance spectra from the multispectral image sets, the MiniMacbeth target was also measured with the ilPro at $10 \mathrm{~nm}$ intervals from 380 to $730 \mathrm{~nm}$. The results were in good correspondence with the corrected camera responses for all four rows of the MiniMacbeth target (Fig. 8). The camera values at $400 \mathrm{~nm}$ were not reliable because the very low power of the lamp in this waveband required long integration times for image capture, with consequent low signal-to-noise ratio. The CIE $L * a * b *$ values were calculated for each reflectance spectrum, using the CIE $2^{\circ}$ standard observer and illuminant D65, and the mean colour difference over the 24 patches was $\Delta E^{*}{ }_{a b}=3.4$. The maximum error was 6.5 for the fourth patch in the second row (purple).

\section{$4 \quad$ Image Registration}

Because of differences in refractive index of the camera lens for different wavelengths of light, the geometry of the successive images in the multispectral sequence varied from one image to the next, so that no two were in perfect register. The treatment may have radically altered the parchment, moreover, causing substantial distortion to its geometric structure. To make a composite image of all the wavebands, therefore, it was necessary to find a way to put them all into accurate register.
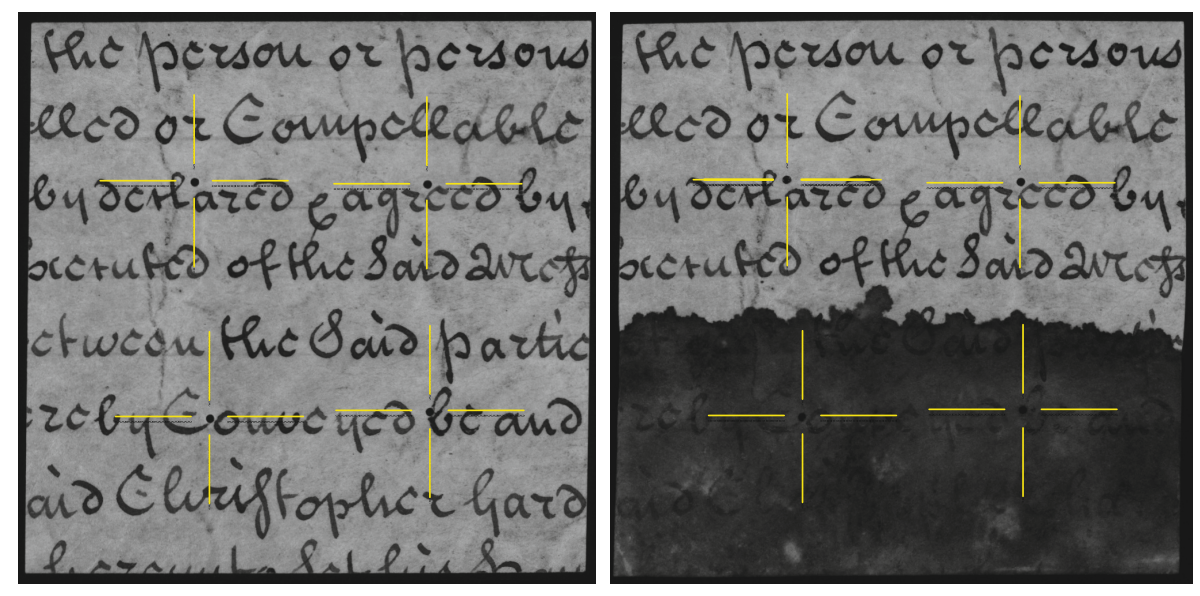

Fig. 9. Images through $600 \mathrm{~nm}$ filter of the parchment sample half-covered by blood, before and after the treatment. The yellow lines indicate the positions of the registration holes. 
The approach taken in this project has been to use the four holes drilled in each parchment sample (Fig. 9) as the anchor points for a projective geometric transform. The image of the sample before treatment taken through the $600 \mathrm{~nm}$ filter was chosen as the reference and all other images, both before and after treatment, were registered to it. A template matching procedure was used to locate accurately the centroid of each of the four holes in each sample. Given an approximate starting position, the algorithm incremented the diameter of a circular anti-aliased template in units of 0.1 pixel (Fig. 12a) over the range 12 to 16 pixels $(1.0$ to $1.3 \mathrm{~mm})$, and the template was successively translated along the $x$ and $y$ axes. At each coordinate position a crosscorrelation was performed between the template and the corresponding section of the image, using the Matlab function corr2 to calculate the correlation coefficient $r$ :

$$
r=\frac{\sum_{m} \sum_{n}\left(A_{m n}-\bar{A}\right)\left(B_{m n}-\bar{B}\right)}{\sqrt{\left(\sum_{m} \sum_{n}\left(A_{m n}-\bar{A}\right)^{2}\right)\left(\sum_{m} \sum_{n}\left(B_{m n}-\bar{B}\right)^{2}\right)}}
$$

This formula has the advantage of being independent of the brightness and contrast of the image $A$ and template $B$, because it takes differences from their respective means and normalises the intensity values. The maximum correlation score over the region was determined, and the centroid of all neighbouring scores above a threshold value gave an estimate of the centre of the hole to sub-pixel accuracy.
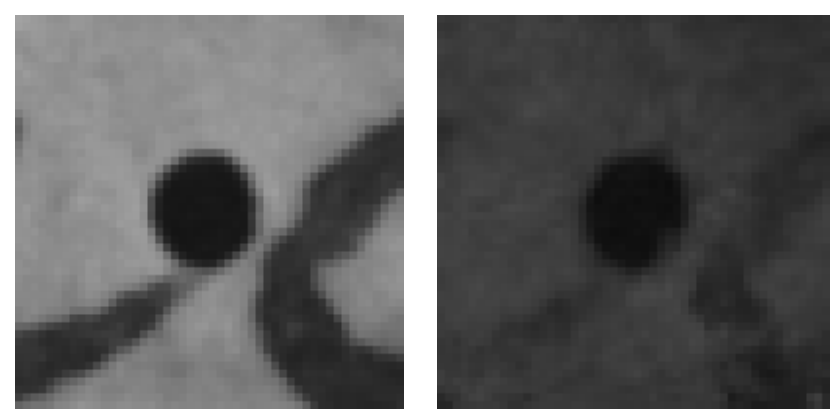

Fig. 10. Enlarged details of the lower left hole in Fig. 9 before and after treatment

Where the hole was located in a region of blank parchment, this procedure worked well and gave a well-defined peak. Where the hole was touching or close to a stroke of a written character, however, the correlation scores could form an elongated ridge (Fig. 11a), which would bias the location of the centroid. Careful tuning of the size of the threshold value to 0.7 and the range of movement of the centre of the correlation region to $13 \times 13$ pixels (approximately the hole diameter) proved to be robust, even when the contrast between the hole and surrounding parchment was reduced by the effect of the treatment. Fig. 10b shows the region after treatment of the sample by blood, causing the ink to be partially dissolved and the image contrast to be reduced. The presence of iron in the blood stains is significant, because it can cause problems in recovering the underlying writing in iron-based ink. In this case, however, the correlation procedure still produced a clearly-defined peak (Fig. 11b). 

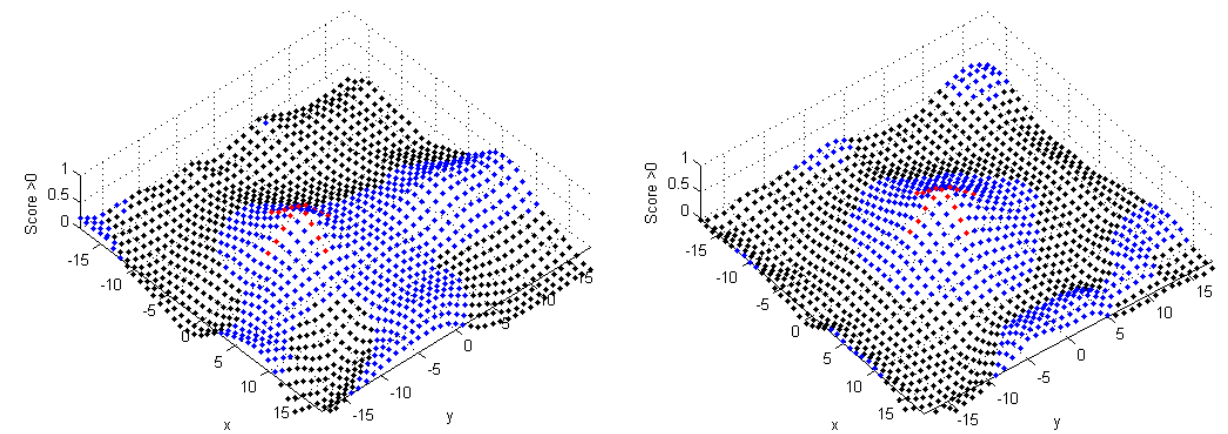

Fig. 11. Correlation scores for the lower left hole before and after treatment (Fig. 10), visualised as surfaces on a pixel grid, showing the peak score at the hole centre, and secondary peaks caused by the ink strokes. Scores $<0$ are represented by black dots, and scores $>0$ by blue dots. The red cross shows the row and column indices of the peak score.
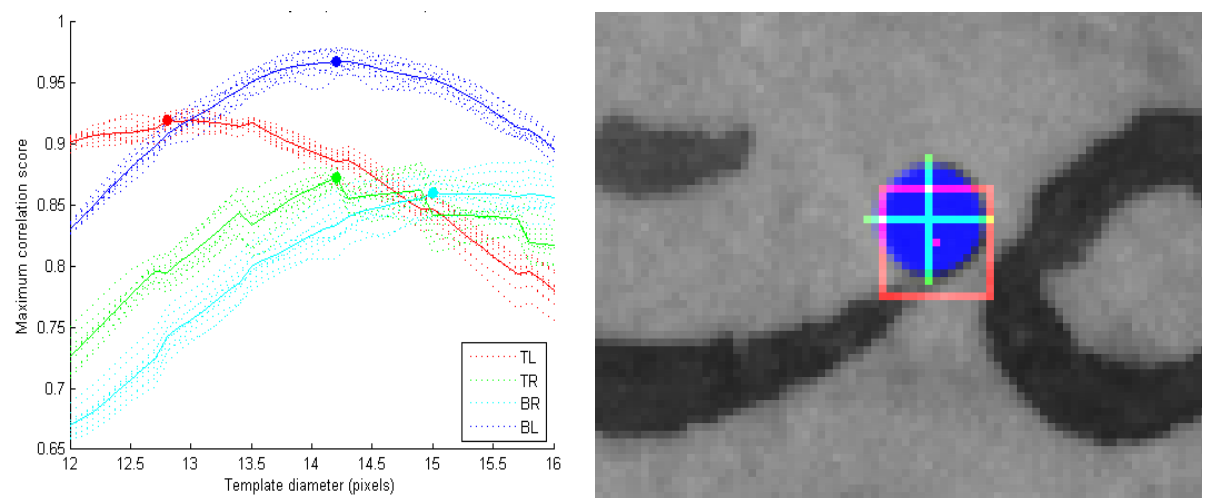

Fig. 12. (left) Maximum correlation score for each hole as a function of template diameter in pixels; (right) centroid (cyan cross) of template position (blue) over image of neighbourhood of lower left hole (same as Fig. 10a). The red square is the range of the correlation region.

The mapping of each image to the reference image is performed by projective mapping, using the Matlab functions maketform and imtransform. In this projective transformation, quadrilaterals map to quadrilaterals and straight lines remain straight. It is implemented as:

$$
\left[\begin{array}{lll}
u^{\prime} & v^{\prime} & w^{\prime}
\end{array}\right]=\left[\begin{array}{lll}
x & y & z
\end{array}\right] \mathbf{T}^{-1}
$$

where $u=u^{\prime} / w^{\prime}$ and $v=v^{\prime} / w^{\prime}$ and $\mathbf{T}^{-1}=\left[\begin{array}{ccc}\mathrm{A} & \mathrm{D} & \mathrm{G} \\ \mathrm{B} & \mathrm{E} & \mathrm{H} \\ \mathrm{C} & \mathrm{F} & \mathrm{I}\end{array}\right],=\frac{\mathrm{A} x+\mathrm{B} y+\mathrm{C}}{\mathrm{G} x+\mathrm{H} y+\mathrm{I}}, v=\frac{\mathrm{D} x+\mathrm{E} y+\mathrm{F}}{\mathrm{G} x+\mathrm{H} y+\mathrm{I}}$

At least four control-point pairs are needed to solve for the nine unknown coefficients of $\mathrm{T}^{-1}$ and these are provided by the $(x, y)$ centroid coordinates of the four anchor points. 
The accuracy of the registration procedure is shown in the false-colour image composite (Fig. 13). The transformed image (red channel) is closely in register with the reference image (blue channel), so that they appear everywhere as a unified magenta.
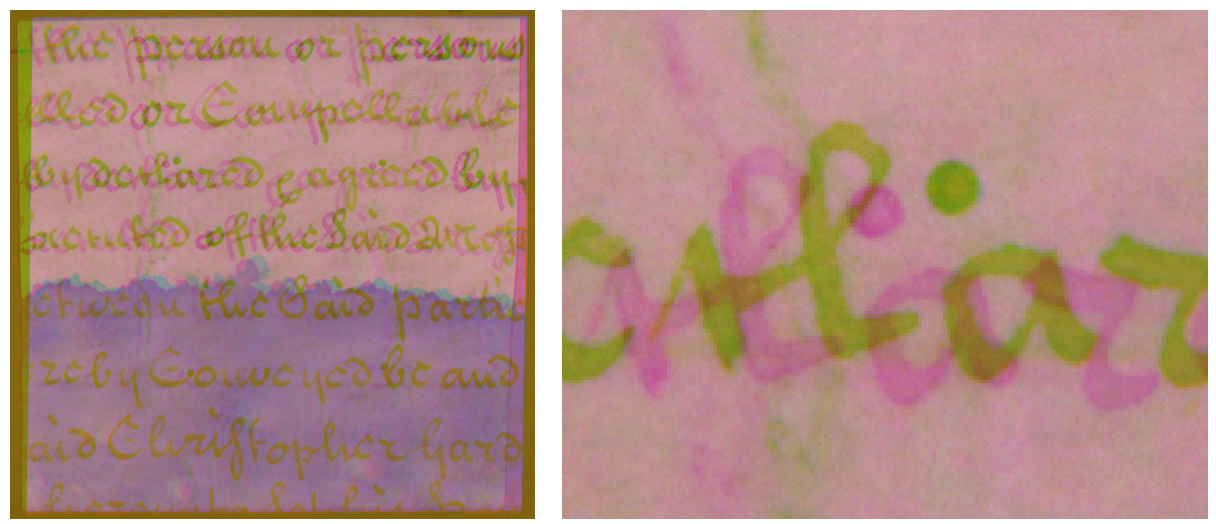

Fig. 13. (left) False-colour composite of reference $600 \mathrm{~nm}$ channel of sample before treatment (blue), $850 \mathrm{~nm}$ channel of sample after treatment (green), and the latter after registration (red); (right) enlarged detail of region around upper left hole

\section{$5 \quad$ Image Analysis}

When all of the image channels for the sample before and after treatment have been accurately registered, it is possible to compare the effect of the treatment on the reflectance spectrum at any pixel position. Fig. 14 shows the effect of the treatment by blood on the parchment and the iron gall ink in locations close to the bottom left hole (Figs. 9 and 10). The spectra are plotted for all nine pixels in a $3 \times 3$ region around the selected coordinates, together with the mean in green (before) and red (after). The blood clearly affected the colour of the parchment, reducing its reflectance from about 0.5 to less than 0.1 in the short wavelengths, rising to about 0.4 at long and NIR wavelengths, giving a dark red colour. The ink spectrum was apparently not much changed (Fig. 14 right), except at wavelengths greater than $850 \mathrm{~nm}$, but comparison with the figure at the left shows that the result is not much different from the blooded parchment spectrum, because most of the ink has been dissolved.
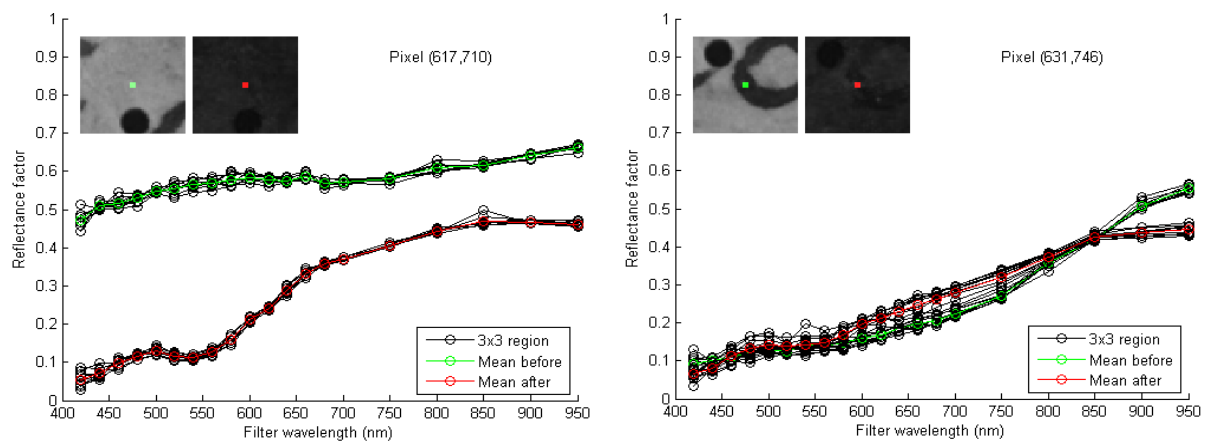

Fig. 14. Reflectance spectra before and after treatment for (left) parchment and (right) ink 
Because the reflectance spectrum can be estimated from the multispectral image set, the appearance of the parchment under any illumination source of known spectral power distribution can be predicted. Colour renditions of the sample after treatment were reconstructed from the registered image channels, using the wavelengths 400700 in the visible spectrum, interpolated to $5 \mathrm{~nm}$ intervals, with the CIE $2^{\circ}$ Standard Observer and four common illuminants: cool daylight (D65), incandescent (A), triband fluorescent (F11), and light-emitting diode (LED):

$$
X=k \sum_{400}^{700} S(\lambda) \cdot R(\lambda) \cdot \bar{x}(\lambda) \cdot \Delta \lambda
$$

where: $X$ is a scalar stimulus value, $S(\lambda)=$ power of source at each wavelength $\lambda$, $R(\lambda)=$ surface reflectance, $\bar{x}(\lambda)=$ standard observer tristimulus function, $\Delta \lambda$ is the wavelength interval $(5 \mathrm{~nm})$, and $k$ is a normalising factor. Similar equations apply for $Y$ and $Z$, corresponding to $\bar{y}(\lambda)$ and $\bar{z}(\lambda)$.
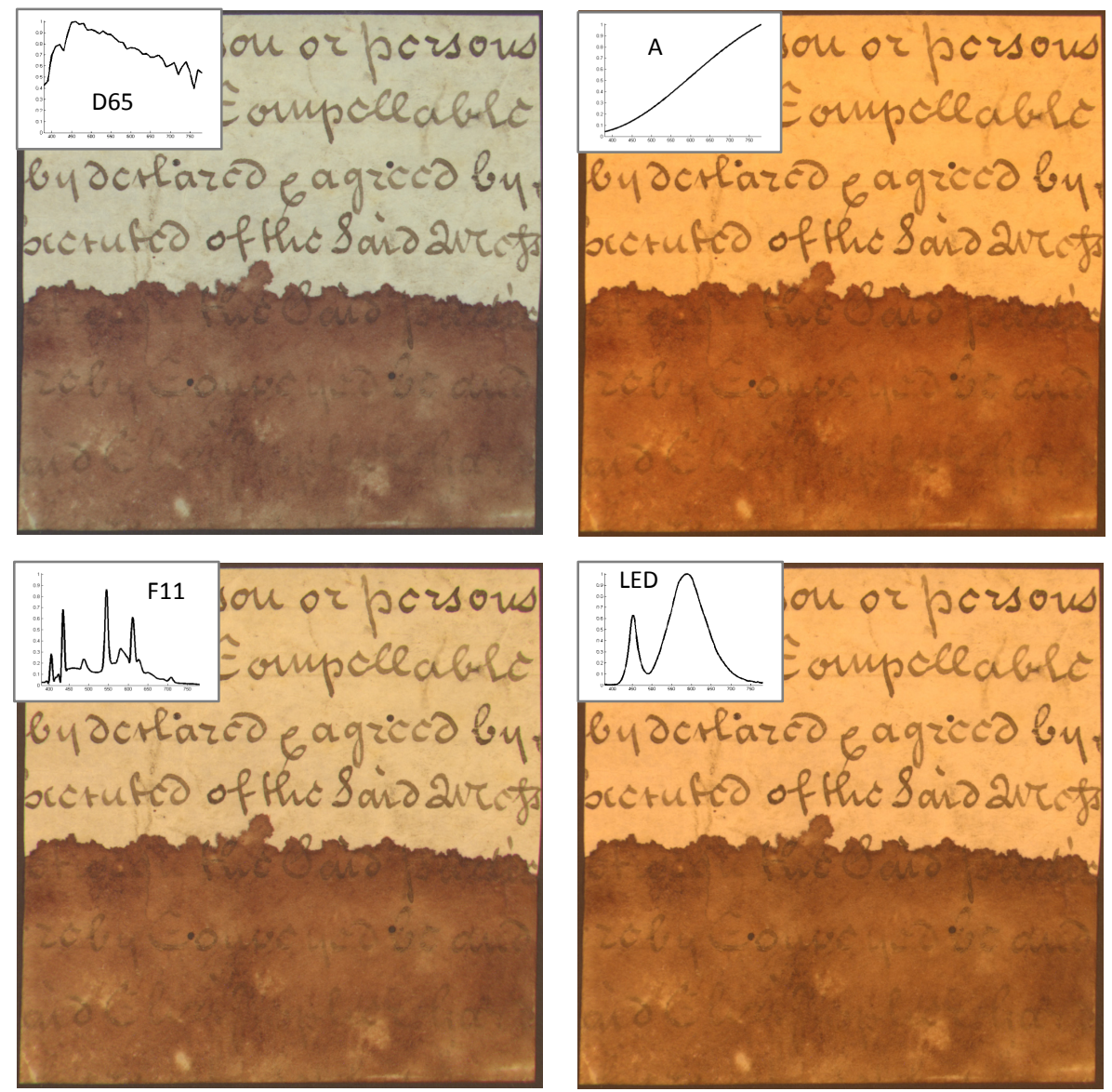

Fig. 15. Colour image reconstruction of sample after treatment under daylight (D65), incandescent, fluorescent and LED sources, with respective spectral power distributions (inset) 
The results, converted from XYZ tristimulus values to sRGB (assuming a D65 display white point), are shown in Fig. 15. The daylight gives a cool bluish appearance, with the parchment almost grey, whereas the incandescent light gives a very warm appearance, with the parchment orange and the blood a rich tomato red. Both the fluorescent and LED sources, which have lower colour rendering indices, exhibit a pink cast, but make the blood look more brown than red.

\section{Discussion}

Multispectral imaging enables the reflectance spectrum of the surface to be estimated at every pixel position in the image. This makes it a much richer representation than the usual trichromatic RGB image, and thereby enables a deeper level of analysis. For this project in particular, it enables the effect of the treatment to be characterised in terms of its effect on the reflectance spectrum of the materials.
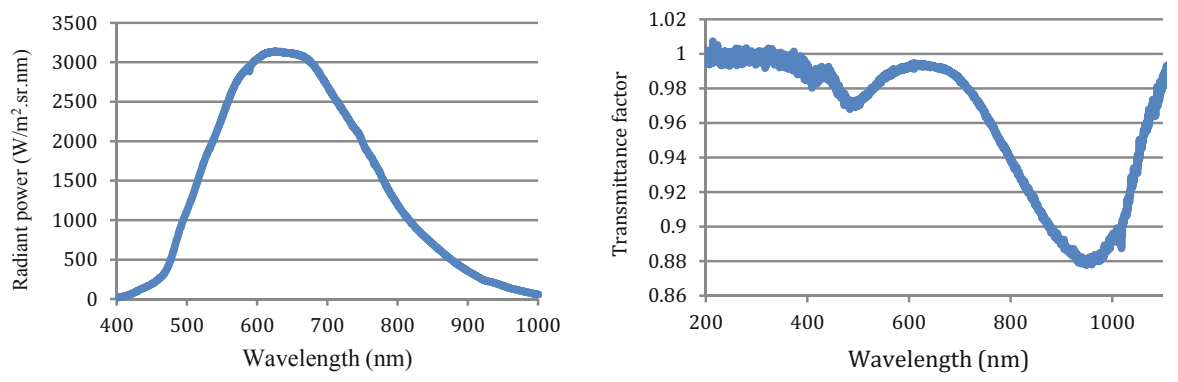

Fig. 16. (left) Spectral power distribution of the tungsten-halogen lamp on copystand; (right) Transmittance factor of anti-reflective glass sheet placed over samples

The tungsten-halogen lamps used on the copystand for illuminating the parchment samples produced a continuous spectral power distribution (Fig. 16a), but suffered from low power at short wavelengths, especially at $400 \mathrm{~nm}$. Each sample was covered by a sheet of $3 \mathrm{~mm}$ glass with an anti-reflective coating, in order to hold it flat on the baseboard of the copystand during photography. This was particularly important for the samples after treatment, as they were inclined to curl. The transmittance factor of this glass was measured (Fig. 16b) and throughout the visible spectrum was greater than 0.97 , although it dropped to 0.88 in the IR. The method of using a white reference for each filter wavelength was effective because it compensated not only for spatial variations in the illumination and lens vignetting, but also for variations in the power of the light source, transmittance of the filter glass and sensitivity of camera.

The method for registering the images in this study makes use of four circular holes drilled in each parchment sample. This proved to be effective in registering all image sets both before and after treatment to a common geometric grid, but was limited in two ways: (a) the accuracy of locating the hole centroids depends on their shape, the image contrast, and whether there are any adjacent ink strokes. Iterative techniques with radiometric adaptation would give greater precision in the centroid coordinates [16]; (b) the projective transform with coefficients derived from the four 
hole centroids can correct for translation, scaling, rotation and perspective changes, but cannot correct for non-linear distortion of the parchment which results from some treatments such as heat and humidity. In general it produced better results for the central square image area bounded by the four holes, and worse results outside this area where the transform was extrapolating. Better results would be obtained by the detection of additional image features, including ink and other markings, over the whole area, then employing a 'rubber sheet' mapping technique [17].

A better solution would be to determine a consistent feature set over the entire parchment area and to use this for registration of the image set. Whilst such a feature set is highly dependent on the image texture, a spatially dense feature set would support the use of local coordinate transformations, avoiding extrapolation at image edges and ensuring that localised text is correctly registered throughout. The method would have the added benefit of correcting for radial geometric shifts in the imagery due to differing refraction of the recording optics

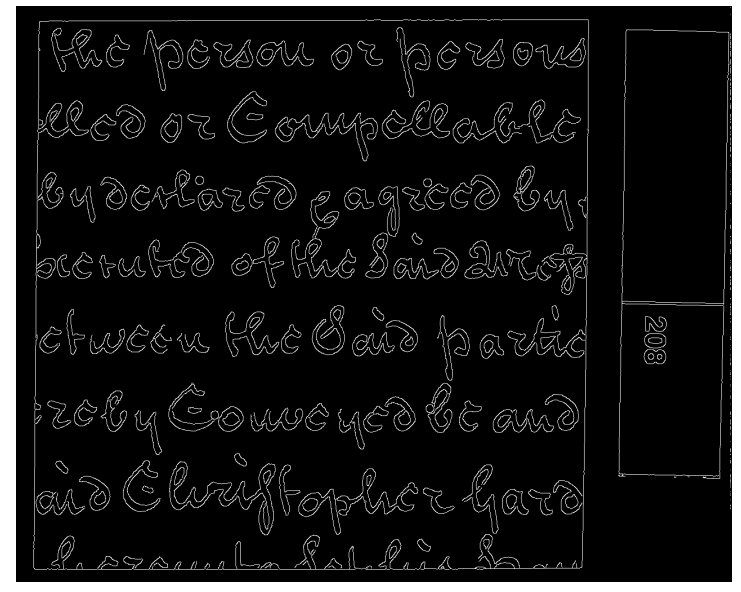

Fig. 17. Edge extraction of text provides a rich feature set for subsequent registration of images with wavelength. The example in Figure 17, which has been processed using image dilation and erosion followed by a Canny edge detector, shows how key corresponding points for subsequent correlation might be seeded from the text features. The clarity of the extraction also demonstrates the merit of matching between image channels using edge registration methods.

A typical problem where a degraded parchment is subjected to analysis is that its original state (both text and material) is not known, and so assumptions must be made in guiding the digital restoration. Our dataset of the parchment samples before treatment provides a 'ground truth' for analysis of the effectiveness of image processing algorithms in restoring images of the degraded samples. Our dataset of the samples after treatment shows exactly the effect of the physical changes on the reflectance. Analysis of multispectral images of scraped or mechanically damaged samples enables us to identify even faint traces of ink. It also enables the discrimination of inks similar in appearance, and the recovery of writing from darkly stained parchment and from charred and burned fragments.

As a result of this project we have sets of multispectral images showing both the initial and degraded state of a manuscript for each of a series of treatments typical of the degradation of real parchments encountered in practice. These image sets will be fully documented and released publicly as a resource for the research community. We offer these as a guide for conservation, a resource for quantitative evaluation of image processing algorithms for information recovery, and a stimulus for other research activities in conservation, image processing, computer graphics, and archaeometry. 
Acknowledgements. This research was funded by EPSRC in the UK under Grant number EP/F01208X/1. We thank the London Metropolitan Archive for providing the original parchment.

\section{References}

1. Terras, M., Robertson, P.: Image to Interpretation: An Intelligent System to Aid Historians in Reading the Vindolanda Texts, p. 252. Oxford University Press, Oxford (2006)

2. Chabries, D.M., Booras, S.W., Bearman, G.: Imaging the past: Recent applications of multispectral imaging technology to deciphering manuscripts. Antiquity 77(296), 359 (2003)

3. Salerno, E., Tonazzini, A., Bedini, L.: Digital image analysis to enhance underwritten text in the Archimedes palimpsest. Intl. J. Doc. Analysis and Recognition 9(2), 79-87 (2007)

4. Tanner, S., Bearman, G.: Digitizing the Dead Sea Scrolls. In: Proc. IS\&T Archiving Conf., pp. 119-123 (2009)

5. Reed, R.: Ancient Skins, Parchments and Leathers. Seminar Press, London (1972)

6. Clarkson, C.: Rediscovering Parchment: the nature of the beast. The Paper Conservator 16(1), 5-26 (1992)

7. Larsen, R.: Introduction to damage and damage assessment of parchment. Improved damage assessment of parchment (IDAP): assessment, data collection and sharing of knowledge. European Commission, Directorate-General for Environment, 17-22 (2007)

8. Giacometti, A., Campagnolo, A., MacDonald, L., Mahony, S., Terras, M., Robson, S., Weyrich, T., Gibson, A.: Documenting Parchment Degradation via Multispectral Imaging. In: Proc. BCS Conf. on Electronic Imaging and the Visual Arts (EVA), London (2012)

9. Vane, G., Goetz, A.F.H.: Terrestrial imaging spectroscopy. Remote Sensing of Environment 24(1), 1-29 (1988)

10. Landgrebe, D., Chen, C.: Information Extraction Principles and Methods for Multispectral and Hyperspectral Image Data, pp. 3-38. World Scientific Pub., River Edge (1999)

11. Hardeberg, J.Y.: Acquisition and Reproduction of Color Images: Colorimetric \& multispectral approaches, Dissertation.com (2001)

12. Saunders, D., Cupitt, J., Padfield, J.: Digital imaging for easel paintings. In: MacDonald, L.W. (ed.) Digital Heritage: Applying Digital Imaging to Cultural Heritage, ch. 19, pp. 521-548. Elsevier, Oxford (2006)

13. Attas, E.: Enhancement of Document Legibility Using Spectroscopic Imaging. Archivaria 57, 131-146 (2004)

14. Bianco, S., Colombo, A., Gasparini, F., Schettini, R., Zuffi, S.: Applications of Spectral Imaging and Reproduction to Cultural Heritage. In: Stanco, F., Battiato, S., Gallo, G. (eds.) Digital Imaging for Cultural Heritage Preservation: Analysis, Restoration, and Reconstruction of Ancient Artworks (Digital Imaging and Computer Vision). CRC Press, Boca Raton (2011)

15. Bearman, G.H., Spiro, S.I.: Archaeological Applications of Advanced Imaging Techniques. The Biblical Archaeologist 59(1), 56-66 (1996)

16. Baltsavias, E.: Multiphoto Geometrically Constrained Matching. D.Sc. Thesis, ETH Zurich, p. 73 (1991)

17. White, M.S., Griffin, P.: Piecewise Linear Rubber-Sheet Map Transformation. Cartography and Geographic Information Science 12(2), 123-131 (1985) 